\title{
Research and Construct of Low Power, Excessive- Velocity Comparators using Cmos Generation with Low Deliver Voltages for Adcs
}

\author{
K.Saishiva, P.Dass
}

\begin{abstract}
The evaluation of many comparator outcomes for the given requirement having excessive velocity using analog to digital converters is growing, this are controlled using CMOS comparators which are successful when delivering the low voltage with high efficiency. The comparators are primary part of numerous simple to computerized converters. The prerequisite for low-control, rapid simple to advanced converters is increasing. Thus comparators are generally utilized in the present innovation because of its quick operational speed and high precision. The quickly developing versatile gadget requires low power and high operational capacities which should be improved. A concise investigation of traditional double tail voltage comparator is done and dependent on that, a low power and region productive comparator is displayed. Another comparator is planned so as to decrease the postponement of ordinary comparators and diminish the power utilization of the gadget. Furthermore, the Reproduction is finished by Leather Treated Simple Plan Condition. At last we study about conventional dual tail voltage comparator which is done based on low power and area efficient comparator. In this simulation of proposed comparator is occurs a 180nm CMOS technology its consumes the power of $69 \mu \mathrm{W}$ at $1.2 v$ Ac power supply voltage.
\end{abstract}

Keywords-COMS Comparators, Analog to Digital converters, latch.

\section{INTRODUCTION}

The advanced converter (ADC) is a fundamental constructing block run VLSI IC shape industry. Which might be use inside the extraordinary interchanges tools and biomedical packages requests for instant circuits and different important structure squares which require much less electricity for its operation. Henceforth the aggregate of diverse realistic ADCs more and more common place device. But only ADCs which are having less transistor measurement, low power necessity and with speed operation are reasonable to use in IC plan agencies. Alongside those traces, some other ADC which calls for less strength in voltage supply giant factor. The comparator is an vital rectangular within the planning of maximum huge speed ADCs. Comparators which are used in interface area of analog to make it advance in crucial cutting edge conversation and biomedical frameworks. regular for an $\mathrm{ADC}$, for example, excessive desires and excessive exactness is direct manipulate by means of the comparator.

Revised Manuscript Received on August 14, 2019.

K.Saishiva,UG student, Department of Electronic and Communication Engineering, Saveetha School of engineering, Saveetha Institute of Medical and Technical Sciences, Chennai, TamilNadu, India (email: kumaravelsaishiva@gmail.com)

P.Dass, Assistant Professor, Department of Electronic and Communication Engineering, Saveetha School of engineering, Saveetha Institute of Medical and Technical Sciences, Chennai, TamilNadu, India (email: dasseie@gmail.com) in this manner, high-performance comparator that is prepared for intensifying little facts voltage to an high voltage is used by designing this type of comparators to achieve more speed in given innovation with supplying low voltage.

The comparator is widely used device next to op- amp in the electronics devices. A comparator with high voltage, low power consumption has given complete delay analysis for low power applications. A low power CMOS comparator is designed for signal processing applications using bipolar CMOS technology.

\section{EXISTING SYSTEM}

In this, the comparator electricity intake and decreased delay time accordingly operation. however unmarried discharge route via tail transistor for each stage in addition to the go inverter where in stage to keep enter inversion region with pair of transistors using small modern-day and with the latch calls for massive cutting-edge in fast generation to permit coupled inverter level which is not applicable at first discharge course and the performance is limited using tail transistor. Therefore, comparator using one-of-a-kind cutting-edge discharge direction with differential pairs and the cross coupled inverter latch is used in other direction. Also the comparator will face strength dissipation because synchronizing of clocks will lead to decrease in velocity. Comparator with dynamic latch is supplied for better results in accuracy, energy intake and finally it delivers a some voltage when compared to other comparators.

\section{PROPOSED SYSTEM}

In proposed system, we add some transistors to conventional dual tail comparator to improve the performance of dual tail comparator. The proposed comparator is added with two transistors and gated nodes. During initial phase, the transistor saves static power loss by providing strong isolation between VDD to GND. During decision making phase, unless one of the transistor turns OFF and another transistors restricts the flow charge from VDD to GND in order to reduce consumption of power in the circuit. Hence the consumption of power in the comparator is reduced. As the additional modification we reduce two transistors, in the absence of these two transistors, the charge flow very quickly from VDD to GND and it increases in comparing the voltages between nodes of

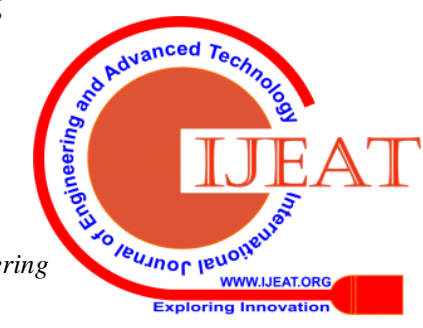




\section{RESEARCH AND CONSTRUCT OF LOW POWER, EXCESSIVE-VELOCITY COMPARATORS USING CMOS GENERATION WITH LOW DELIVER VOLTAGES FOR ADCS}

$\mathrm{fn} / \mathrm{fp}$. Hence the latch regeneration speed of the comparator is increased.

\section{CONVENTIONAL COMPARATOR}

Mostly, conventional comparators are used in ADCs convertors due to static power consumption is 0 and also having high value in input impedance. At first phase clock is given as zero volts $\mathrm{CLK}=0 \mathrm{~V}$, transistor Nmos and Mtail is kept in OFF state. Both transistors M7 and M8 of Pmos force the output node Out+ and Out- to supply the voltage VDD in pre phase. During the evaluation phase when clock equals to VDD then both Pmos transistors M7 and M8 goes OFF state and Nmos transistor is kept in ON state. Finally, the output node voltages which are charged to VDD are began to discharge which depends on input voltages Vin and Vref.

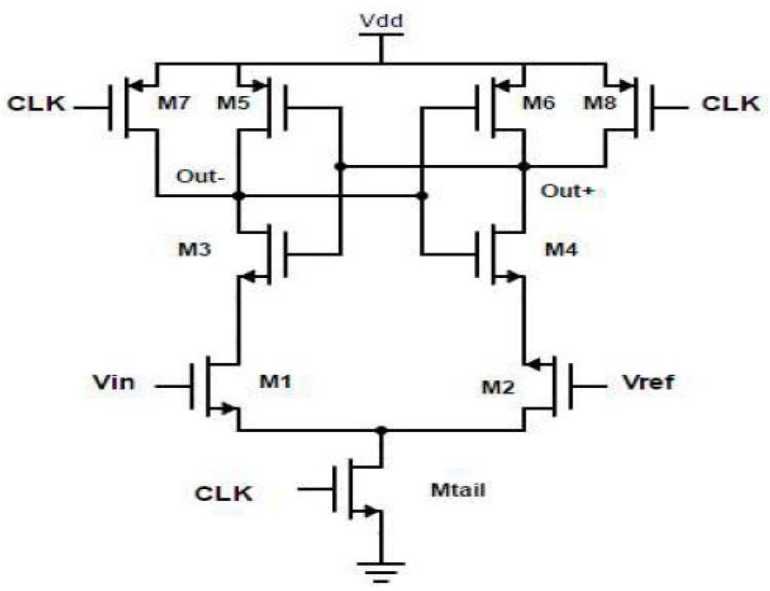

Fig 1: Conventional Comparator.

If Vref is greater than Vin, then Out+ node releases faster charge composition to Out- node. According to that condition before the Out- voltage drops, Out+ node is dropped to Vth value. Where the Pmos transistor M5 and latch could be $\mathrm{ON}$ and start the regeneration of the latch with go coupled inverter pairs of M3, M5 and M4, M6. Finally, when the Out- node is charged to VD then Out+ is dropped to zero. If Vref is lesser than Vin then the circuits operation is done vice versa. While using this structure we get benefits of full output swing, operation is not stopped during noise and mismatch. And the drawback for this structure is, for using the several transistors we need to give high voltage so they delay in time,

\section{DOUBLE TAIL COMPARATOR}

In this process, the comparator uses contemporary discharge method for one of the pairs of input and other course for cross coupled latch. By using this we can say it is a dual tail comparator. This comparator gives a correct voltage when compared to standard comparators. For heavy current and fast latching operation, the is done through Pmos transistor Mtail2 and it is used widely nowadays. At the starting of the operation we have $\mathrm{CLK}=0 \mathrm{~V}$ and the transistors Mtail1,Mtail2 goes OFF and at the same time Pmos transistors M3,M4 goes ON. And this two transistors compelled with the nodes of Fi- and Fi+ with vdd due to MR1 and MR2 of Nmos transistors are in active state. Finally, the output node voltage goes to zero. At the stage of evaluation when the clock is equal to VDD then the transistors Mtail1 and Mtail2 goes ON. Then the transistors of Pmos goes to OFF stage and the nodes of voltage Fi- and $\mathrm{Fi}+$ are started to drop.

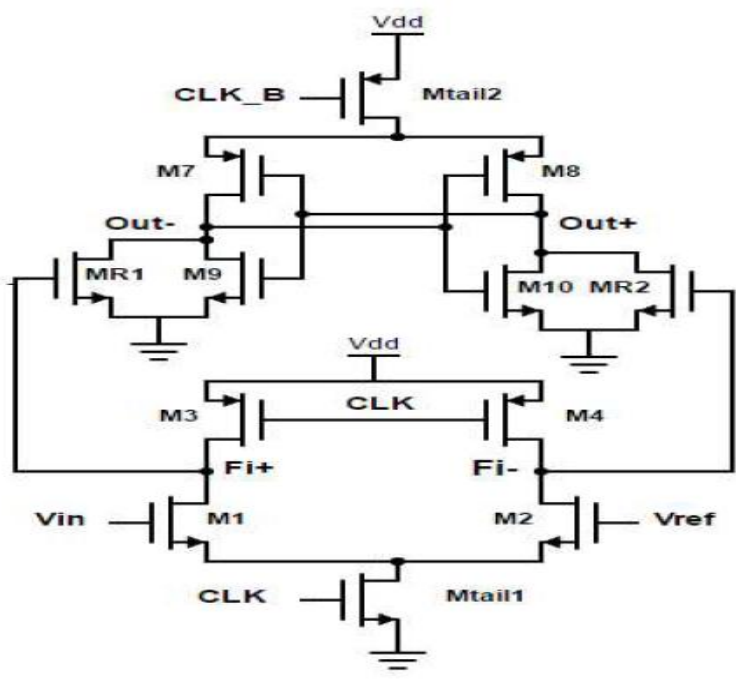

Fig 2: Double tail comparator.

The primary down side of the shape is find of Clock and clock_ B which desires robust timing access. By using the latch degree, the extraordinary input voltage is changed at a particular given time. In the area of Clock_B, invertor is used instead of that so it becomes the input for inverter. Then to gain heavier load Pmos transistor is forced to big size. The extended power of dissipation in comparator is done when Clock_B leads clock and is accelerated to put off. Short circuit route is created in the case of Mtail2 to MR1/MR2 .

\section{DUAL TAIL COMPARATOR}

In this process, the comparator uses two process that is initial and decision making phases. During initial phase $\mathrm{CKL}=0$, hence the nodes fn, fp are attached to VDD which makes transistors (MR1,MR2) to conduct (ON). Due to this, both the final nodes are attached to ground. During the phase of decision making when CLK is equal toVDD, the charge stored at nodes of $\mathrm{fn}$, fp begins to discharge via transistors M1/M2-Msw1/Msw2-Mtail2 to the ground according to the potential applied across the input transistor, suppose voltage across the input INP>INN. The transistor MR2 switched OFF earlier than MR1 and hence the latch generate by the cross coupled inverter which pulls the node Outp towards VDD and the node Outn remains connected to ground. When INN>INP, the working of the circuit is made vice-versa. 


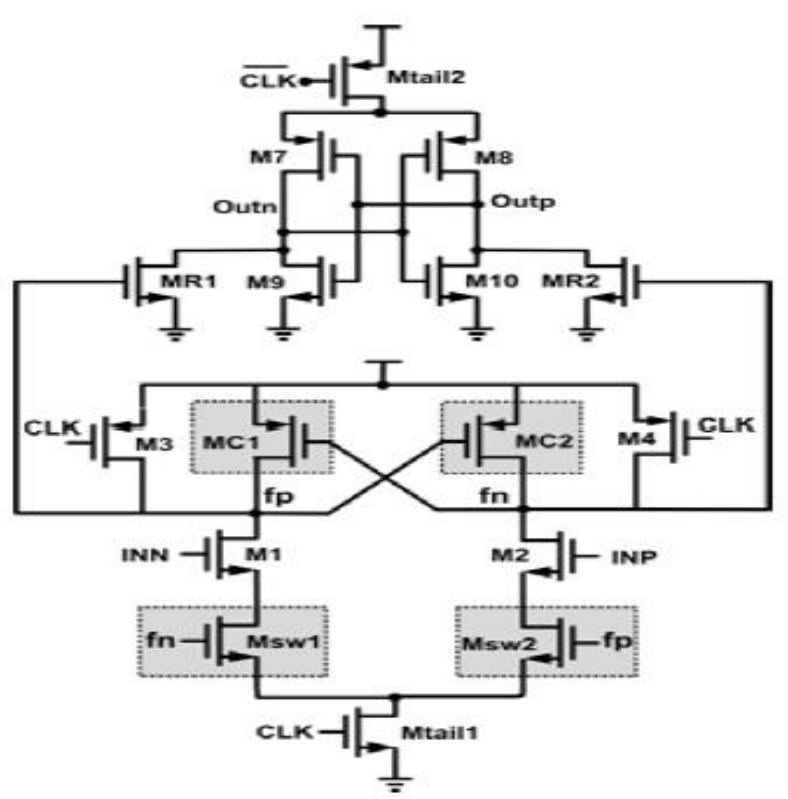

Fig 3: Duel Tail Comparator

The cross coupled inverter proposed for latch regeneration is complex architecture and hence consumes more power for comparison operation. The new latch architecture need to be designed which performs comparison operation with less power.

Waiting of comparator is calculated in two parts, latch detection delay ( $\mathrm{t} 0$ ) and latch regeneration delay (latch). The latch detection delay is the time taken by the comparator to turn OFF one of the transistor MR1/MR2. The latch detection delay is greatly dependents on the transistance conductance of the M1/M2 transistors. The latch regeneration delay is the time taken by the comparator to generate latch by the cross coupled inverter. The transistors (Msw1, Msw2) are interdependent to charge potential at nodes fn/fp during decision making phase in order to consume the power low and hence they restrict the flow charge from $\mathrm{fn} / \mathrm{fp}$ nodes to ground. Due to these, the starting voltage difference of nodes $\mathrm{fn}$, fp are reduced. So, they increase in the comparator delay.

\section{IMPLEMENT COMPARATOR}

To improve the dual tail comparator, a architecture of comparator is proposed by adding few transistors to that comparator. Final design of the comparator is figured below. The proposed comparator consists of addition of M5 and M6 transistors and gated nodes $\mathrm{fn} / \mathrm{fp}$. During initial phase $\mathrm{CKL}=0$, hence the nodes $\mathrm{fn}, \mathrm{fp}$ are fixed to VDD and makes transistors (MR1, MR2) to conduct (ON). Due to this, both the output nodes of transistors are connected to ground. At the similar time, PMOS transistors M5 and M6 are switched OFF to reduce static power loss in the circuit. During the phase of decision making when CLK is equal to VDD, the charge stored at fn and fp nodes which begins to discharge via transistors M1/M2-Mtail2 to the ground according to the potential applied across the input transistor, suppose voltage across the input INP>INN. The transistor MR2 switched OFF earlier than MR1 and hence the latch generate by the cross coupled inverter which pulls the node Outp to VDD and node Outn remains connected to ground. When INN is greater INP, the working of the circuit is vice-versa.

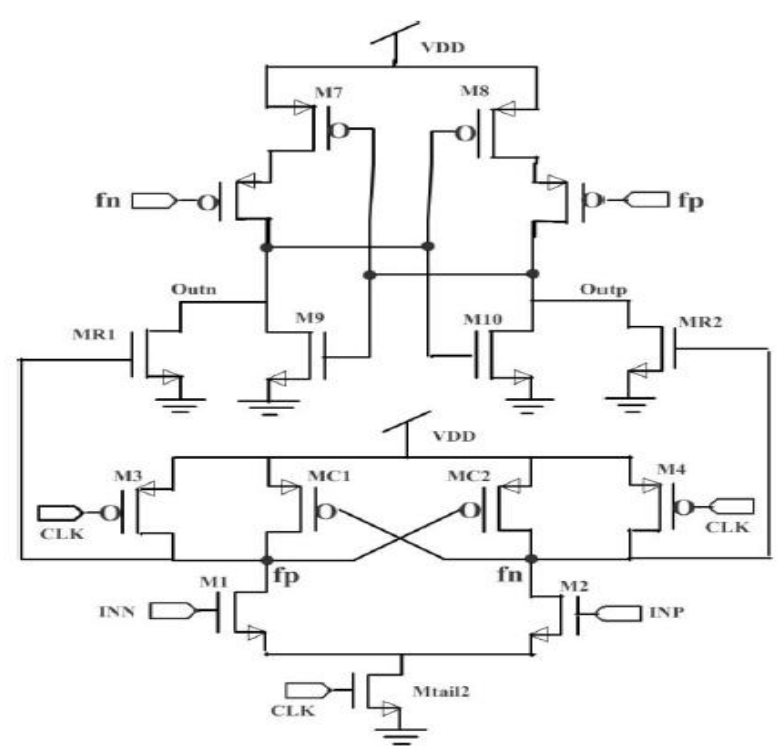

Fig 4: Implement Comparator.

Proposed comparator with addition of M5 and M6 transistors, gated nodes fn/fp is implemented. During initial phase, the transistors M5/M6 save static power loss by providing strong isolation between VDD to GND. During decision making phase, unless one of the transistor form MR1/MR2 turns OFF, transistors M5/M6 restricts the flow charge from VDD to GND in order to low the power consumption of the circuit. Hence, the overall comparators power consumption is reduced. Shows the proposed comparator without Msw1 and Msw2 transistor. In the absence of these two transistors, the charge flow very quickly from VDD to GND and increases the differential voltage difference between $\mathrm{fn} / \mathrm{fp}$ nodes. Hence the latch regeneration speed of the comparator.

\section{SIMULATION \& RESULT}

\section{Existing 1: CONVENTIONAL COMPARATOR}

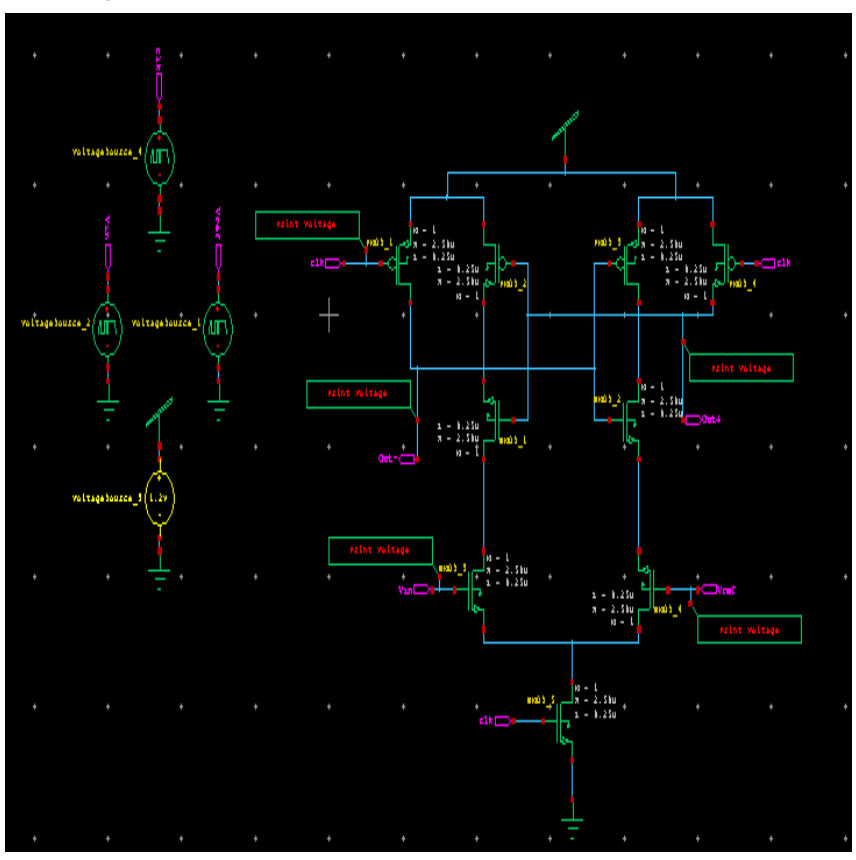

Fig 5: Design For Conventional Comparator 


\section{RESEARCH AND CONSTRUCT OF LOW POWER, EXCESSIVE-VELOCITY COMPARATORS USING CMOS GENERATION WITH LOW DELIVER VOLTAGES FOR ADCS}

In this fig 5 represent the diagram we simulate the low voltage supply in high speed comparator

\section{Output:}

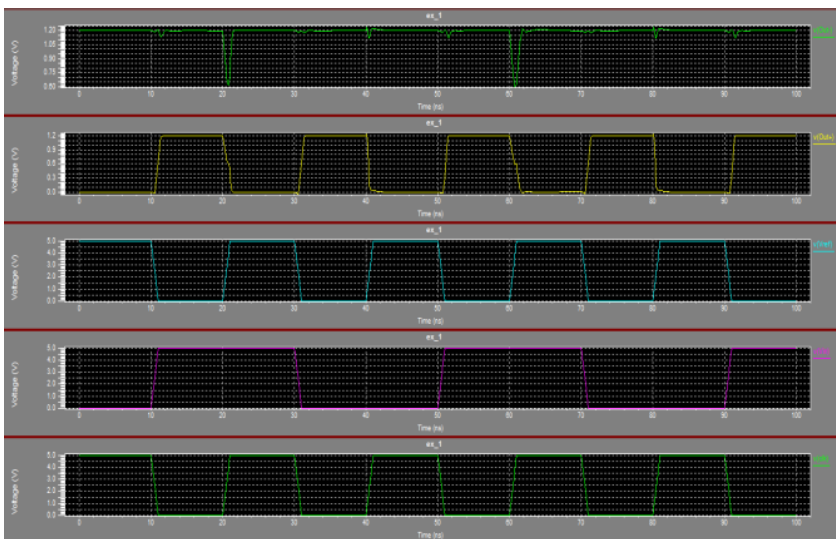

Fig 6: Output The Conventional Comparator

The fig 6 represent output is concave the conventional comparator in this power is $1.2 \mathrm{v}$ will reduce the time in 0.71 seconds in average power consumed in $2.167862 \mathrm{e}-005$ watts

Timing and power analysis:

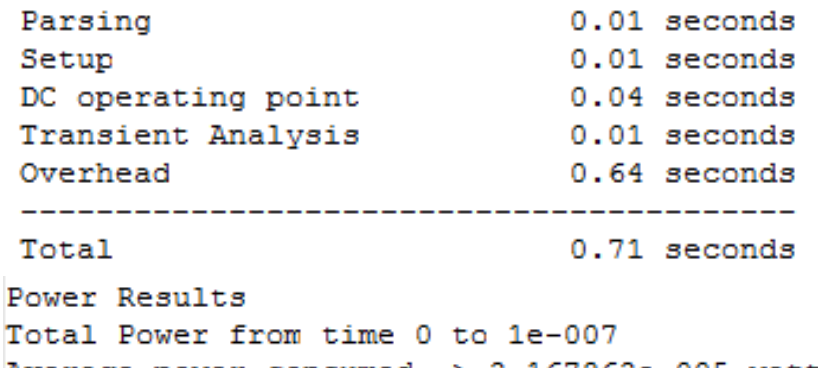

\section{Existing 2: Dual Tail Comparator}

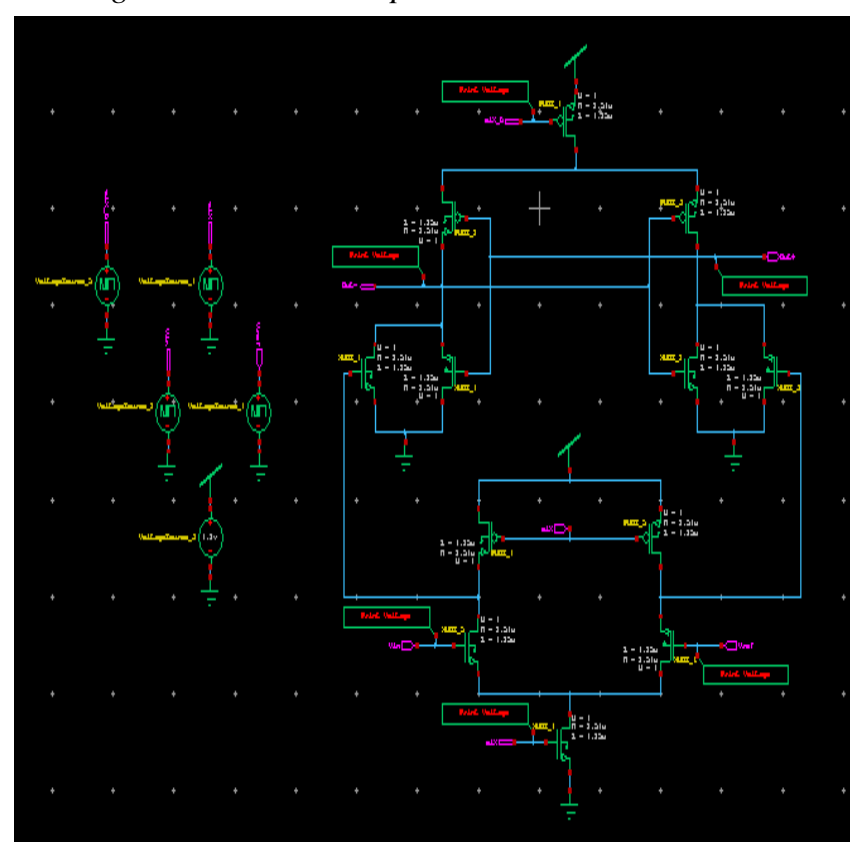

Fig 7: Design for dual tail comparator

In this fig 7 represent the diagram we simulate the low voltage supply in high speed comparator
Output:

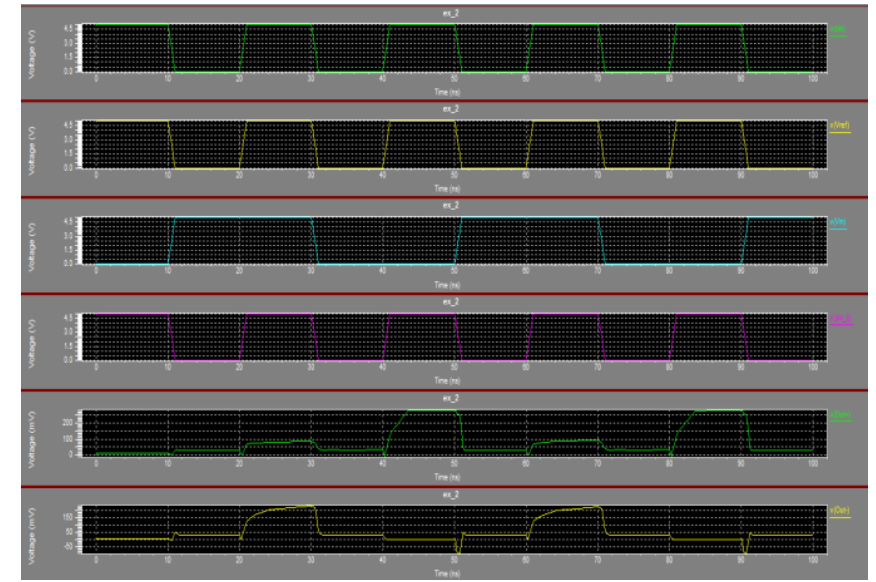

Fig 8: Output Of Dual Tail Comparator

The fig 8 represent output is concave the Dual tail comparator in this power is $1.2 \mathrm{v}$ will reduce the time in 0.86 seconds in average power consumed in $6.893573 \mathrm{e}-005$ watts

Timing and power analysis:

$\begin{array}{ll}\text { Parsing } & 0.01 \text { seconds } \\ \text { Setup } & 0.01 \text { seconds } \\ \text { DC operating point } & 0.00 \text { seconds } \\ \text { Transient Analysis } & 0.01 \text { seconds } \\ \text { Overhead } & 0.83 \text { seconds } \\ \text { - } & 0.86 \text { seconds }\end{array}$

Power Results

Total Power from time 0 to $1 e-007$

Average power consumed $\rightarrow->6.893573 e-005$ watts

Existing 3: DUAL TAIL COMPARATOR

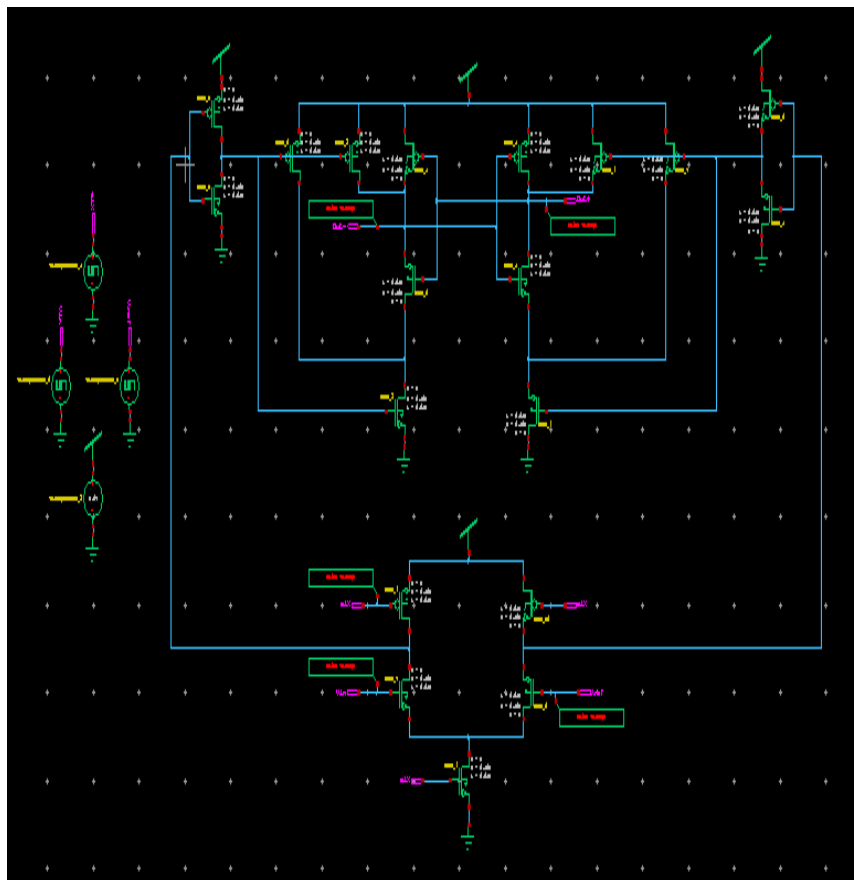

Fig 9: Dual Tail comparator

In this fig 9 represent the diagram we simulate the low voltage supply in high speed comparator

Published By:

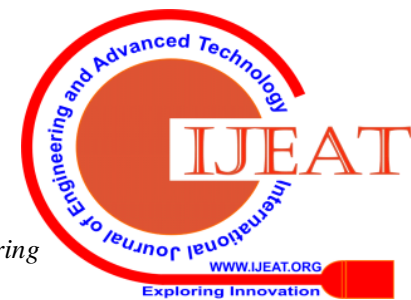




\section{Output:}

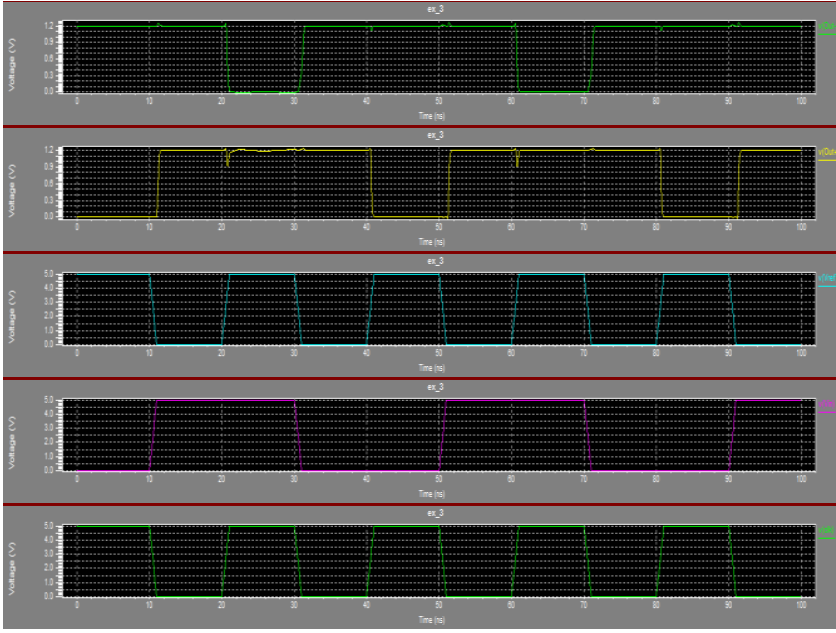

Fig 10: Output Of Dual Tail Comparator

The fig 10 represent output is concave the Dual Tail Comparator in this power is $1.2 \mathrm{v}$ will reduce the time in 0.61 seconds in average power consumed in $2.228750 \mathrm{e}-005$ watts

Timing and power analysis:

\begin{tabular}{|c|c|}
\hline Parsing & 0.01 seconds \\
\hline Setup & 0.01 seconds \\
\hline DC operating point & 0.13 seconds \\
\hline Transient Analysis & 0.03 seconds \\
\hline Overhead & 0.43 seconds \\
\hline ---------- & ----ー-ー--ー--- \\
\hline Total & 0.61 seconds \\
\hline
\end{tabular}

Existing 4: Implement comparator:

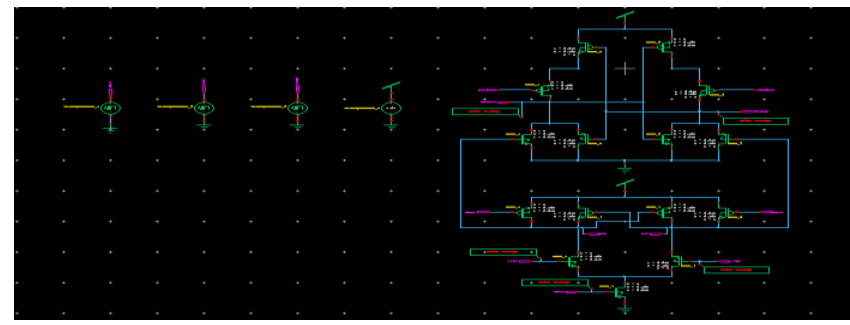

Fig 11: Implement comparator

\section{OUTPUT:}

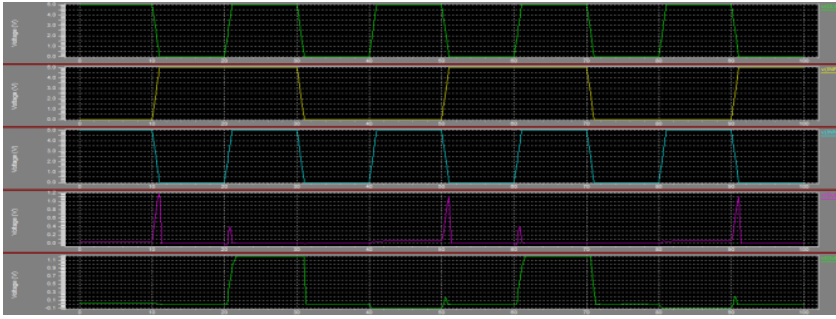

Fig 12: Output Of Implement Comparator

The fig 12 represent output is concave the Implement Comparator in this power is $1.2 \mathrm{v}$ will reduce the time in 0.17 seconds in average power consumed in 1.104854e-004 watts
Timing and power analysis:

$\begin{array}{lr}\text { Parsing } & 0.01 \text { seconds } \\ \text { Setup } & 0.01 \text { seconds } \\ \text { DC operating point } & 0.01 \text { seconds } \\ \text { Transient Analysis } & 0.02 \text { seconds } \\ \text { Overhead } & 0.12 \text { seconds } \\ ------17 \text { seconds } \\ \text { Total } \\ \text { Power Results } \\ \text { Total Power from time 0 to 1e-007 } \\ \text { Average power consumed }->1.104854 \text { - } 004 \text { watts }\end{array}$

\section{CONCLUSION}

The overall performance of ADC is taken through comparator during power consumption. Every parameters are similarly vital, however, between these two parameters tradeoff is done. The whole evaluation of dynamic comparator having traditional twin tail are completed. Based on these comparator, a new type of comparator design is proposed which suggests higher performance than the previous one. The strength consumption of new comparator could be very low in evaluation with the traditional twin tail comparator. The proposed comparator is comes with boom within the latch detection put off because of the addition of M5/M6 transistor. The simulation of the circuits is accomplished by way of Tanner EDA simulation tool the use of CMOS procedure. on this proposed diagram the electricity reduced in $1.104854 \mathrm{e}-004$ watts.

\section{REFERENCES}

1. "Analysis and Design of aLow Voltage Low-Power Double-Tail Comparator", SamanehBabayan-Mashhadi and Reza Lotfi.

2. "Design of efficient Double Tail Comparator for Low Power",SiddharthChaudhari and Mahesh Pawar.

3. "Low Power Comparator Design for SAR-ADC", ChandniSargaiya ,R. S. Gamad.

4. "Low power CMOS comparator using bipolar CMOS technology for signal processing applications", R.Vanitha andS. Thenmozhi.

5. "Design of low leakage current average power CMOS current comparator using SVL technology", SakshiSaxena and Dr. ShyamAkashe.

6. H. Traf, G. Holmbert, and S. Eriksson, "Application of switched current technique to algorithmic DA and ADconverters." IEEE Intl. Symp.Circuits and Systems ISCAS, June 1991, pp. 1549- 1552.

7. D. G. Nairn and C. A. T. Salama "Current-mode algorithmic analog-to-digital converters", IEEE Journal Of Solid-State Circuits, Aug. 1990,Vol. 25, Issue 4, pp. 997 - 1004.

8. S. Babayan-Mashhadi and Reza Lotfi "Analysis and Design of a Low Voltage Low-Power Double-Tail Comparator" IEEE Transactions OnVery Large Scale Integration Systems, Feb. 2013 Vol. 22, Issue 2, pp. 343 $-352$.

9. Siddharth Chaudhari and Mahesh Pawar, "Design of efficient Double Tail Comparator for Low Power." IEEE Conference on Communication and Signal Processing, April 2015.

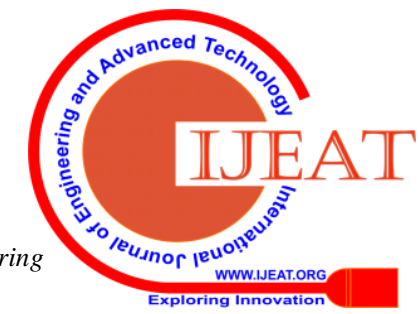


10. Wicht B, Nirschl T, Schmitt-Landsiedel D "Yield and speed optimization of a latch-type voltage sense amplifier." IEEE Journal of Solid-State Circuits, 2004, Vol. 39, Issue 7, pp.1148- 1158.

11. Iffa Sharuddin and L.Lee, "PowerDesign of Low Power and Improved tlatch Comparator for SAR ADC".IEEE Confrerence 2014.

12. H. Jeon and Y. Kim,"A novel low-power, low-offset and high speed CMOS dynamic latched comparator," Analog Integr Circ Sig Process, July. 2011, vol. 70, pp. 337-346.

13. B. Goll and H. Zimmermann "A $65 \mathrm{~nm}$ CMOS comparator with modified latch to achieve $7 \mathrm{GHz} / 1.3 \mathrm{~mW}$ at $1.2 \mathrm{~V}$ and $700 \mathrm{MHz} / 47 ? \mathrm{~W}$ at $0.6 \mathrm{~V}, "$ in Proc. IEEE Int. Solid-State Circuits Conf. Dig. Tech. Papers, Feb. 2009, pp. 328-329.

14. Aparna Lahariya and Anshu Gupta2015, "Design of Low Power and High Speed Dynamic Latch Comparator Using $180 \mathrm{~nm}$ Technology." IEEE International Conference on Signal Processing, Computing and Control, 2015. 